\title{
Off-pump CABG can improve outcomes in patients with CKD
}

The use of off-pump coronary artery bypass grafting $(\mathrm{CABG})$ is associated with improved outcomes compared with on-pump CABG in patients with poor baseline renal function, say the authors of a recent study published in the Journal of the American Society of Nephrology.

\section{These data may help clinicians to identify patients who may be more likely to benefit from an off-pump CABG... 77}

"Despite the fact that the presence of chronic kidney disease (CKD) is an important risk factor for developing cardiovascular events, patients with CKD are systematically excluded from cardiovascular clinical trials, and are often undertreated for cardiovascular disease even though they may have much to benefit," says lead author Lakhmir Chawla from the George Washington Medical Center, Washington DC. "Our data suggest that excluding CKD patients from clinical trials of off-pump CABG may have resulted in an underestimation of the potential benefit for this patient subgroup."

Chawla et al. used the Society of Thoracic Surgery Database to identify a nonrandomized cohort of 742,909 patients who had undergone nonemergency, isolated cases of CABG between 2004 and 2009; 158,561 of these patients received off-pump CABG. The investigators then looked at whether pump status (off-pump versus on-pump) had an effect on the risk of in-hospital death or incident renal replacement therapy (RRT) across different levels of preoperative renal function.

In total, $22.7 \%$ of the cohort had an estimated glomerular filtration rate
(eGFR) of $30-59 \mathrm{ml} / \mathrm{min} / 1.73 \mathrm{~m}^{2}$, and $1.3 \%$ had an eGFR of $15-29 \mathrm{ml} /$ $\min / 1.73 \mathrm{~m}^{2}$; baseline renal function was similar in the two groups.

Using a propensity-weighted analysis to balance demographics and baseline comorbidities across the groups, the researchers showed that off-pump (versus on-pump) $\mathrm{CABG}$ was associated with a slight decrease in the composite of incident RRT or in-hospital death in the overall cohort (1.8\% versus $2.0 \%$, respectively).

Patients with the worst preoperative renal function had the highest incidence of in-hospital death and incident RRT, but the attributable benefit for off-pump (versus on-pump) CABG increased with worsening baseline renal function. The difference in risk for on-pump minus off-pump CABG ranged from 0.05 (95\% CI -0.06 to 0.16 ) per 100 patients with an eGFR of $\geq 90 \mathrm{ml} / \mathrm{min} / 1.73 \mathrm{~m}^{2}$, to $3.66 \mathrm{per}$ 100 patients for those with an eGFR in the range $15-29 \mathrm{ml} / \mathrm{min} / 1.73 \mathrm{~m}^{2}$.

The trend of a greater benefit of offpump (versus on-pump) CABG among patients with the worst preoperative renal function was also shown for both end points individually.

"Our data show that off-pump CABG may benefit patients with baseline CKD and likely offers little benefit to patients with preserved baseline renal function," notes Chawla. "These data may help clinicians to identify patients who may be more likely to benefit from an off-pump CABG as opposed to an on-pump CABG."

Rebecca Ireland

Original article Chawla, L. S. et al. Off-pump versus onpump coronary artery bypass grafting outcomes stratified by preoperative renal function. J. Am. Soc. Nephrol. doi:10.1681/ASN.2012020122 